\title{
Fuzzy Hybrid Filtering Techniques for Removal of Random Noise from Medical Images
}

\author{
R. Marudhachalam \\ Department of Mathematics, \\ Kumaraguru College of Technology, \\ Coimbatore, Tamilnadu, India.
}

\author{
Gnanambal llango \\ Postgraduate and Research Department of \\ Mathematics, \\ Government Arts College (Autonomous), \\ Coimbatore, Tamilnadu, India.
}

\begin{abstract}
Reducing or removing random noise from medical image is a very active research area in medical image processing. In recent years, technological development has significantly improved in analyzing medical images. This paper proposes various fuzzy hybrid filtering techniques for the removal of random noise from medical images, by topological approach. Each of these fuzzy filters, which apply a weighted membership function to an image within a 8-neighbours of a point, is simple and easy to implement. The quality of the noise reduction in images is measured by the statistical quantity measures: Root Mean Square Error (RMSE) and Peak Signal-to-Noise Ratio (PSNR). The performances of these fuzzy filters on images tainted with low, medium and high random noise are compared with various existing filtering techniques.
\end{abstract}

\section{Keywords}

Ultrasound Medical Image, Fuzzy hybrid filters, Random noise, Noise reduction

\section{INTRODUCTION}

Developing tools and techniques to remove random noise (or Gaussian) from medical images plays a very important role. Medical images are often influenced by random noises during image acquisition process. These noises usually affect the visual quality of the original images. Medical image denoising removes noise from the CT, MRI or ultrasound images. Denoising is an essential step that makes diagnosis more efficient for doctors. Several different methods are used to eliminate random noise, based upon different mathematical models of the phenomenon. Noise is usually quantified by the percentage of corrupted pixels. In the literature several fuzzy and non fuzzy filters have been studied for removal of random noise from medical images. In early 1970s median filter has been introduced by Tukey [1]. It is a special case of non-linear filters (non fuzzy filter) used for smoothing signals. Median filter now is broadly used in reducing noise and smoothing the images. In 1993, Kwan and Cai [2,3] developed median filters using fuzzy concepts and also analyzed the performance of various fuzzy filters for noise reduction in images. Nachtegael et al.,[4,5] reviewed fuzzy filters for noise reduction in images and also reported a comparative study of classical and fuzzy filters for noise reduction in 2001. Stefan Schulte et al.,[6] proposed FIDRM(Fuzzy Impulse noise Detection and Reduction method) for reducing all kinds of impulse noise. Gnanambal Ilango and Marudhachalam [7] proposed different types of new hybrid filtering techniques for removal of Gaussian noise from ultrasound medical images. Aneesh Agrawal et al [8] developed an adaptive fuzzy based image filtering techniques for efficient noise reduction in medical images, Gunamani Jena and R Baliarsingh [9] proposed suppression of random valued impulsive noise using adaptive threshold method. This work proposes various fuzzy hybrid filtering techniques for removal of random noise from ultrasound medical images.

This work is organized as follows: In Section 2 basic definitions are given. Section 3 deals with proposed definitions of various fuzzy hybrid filtering techniques for removal of random noise from ultrasound medical images. In Section 4, both quantitative (RMSE \& PSNR) and qualitative comparisons have been provided. Section 5 puts forward the conclusion drawn by this paper.

\section{BASIC DEFINITIONS}

This section presents some general definitions and digital topological results, which will be used along the development of this paper.

\subsection{Digital image}

A digital image [10] is a function $f: Z x Z \rightarrow[0,1, \ldots . . N-1]$ in which $N-1$ is a positive whole number belonging to the natural interval $[1,256]$. The functional value of ' $\mathrm{f}$ ' at any point $\mathrm{p}(\mathrm{x}, \mathrm{y})$ is called the intensity or grey level of the image at that point and it is denoted by $\mathrm{f}(\mathrm{p})$.

\subsection{Neighborhood of a point}

A neighborhood $[10,11]$ of a point $p \varepsilon X$ is a subset of $X$ which contains an open set containing $\mathrm{p}$. It is denoted by $\mathrm{N}(\mathrm{p})$.

\subsection{4-neighbours of a point}

The 4-neighbours [10] of a point $\mathrm{p}(\mathrm{x}, \mathrm{y})$ are its four horizontal and vertical neighbours $(x \mp 1, y)$ and $(x, y \mp 1)$. A point ' $\mathrm{p}$ ' and its 4-neighbours is denoted by $\mathrm{N}_{4}(\mathrm{p})$.

\subsection{Cross neighbours of a point}

The cross neighbours [7] of a point $\mathrm{p}(\mathrm{x}, \mathrm{y})$ consists of the neighbours $(x+1, y \mp 1)$ and $(x-1, y \bar{\mp})$. A point ' $\mathrm{p}$ ' and its cross neighbours is denoted by $\mathrm{C}_{4}(\mathrm{p})$.

\subsection{8-neighbours of a point}

The 8-neighbours [10] of a point $\mathrm{p}(\mathrm{x}, \mathrm{y})$ consist of its 4-neighbours together with its cross neighbours. A point ' $p$ ' and its 8-neighbours is denoted by $\mathrm{N}_{8}(\mathrm{p})$.

\subsection{LT neighbours of a point}

The LT neighbours[7] of a point $\mathrm{p}(\mathrm{x}, \mathrm{y})$ consists of the neighbours $(x-1, y-1)$ and $(x+1, y+1)$. A point ' $\mathrm{p}$ ' and its $\mathrm{LT}$ neighbours is denoted by $\mathrm{L}_{3}(\mathrm{p})$. 


\subsection{RT neighbours of a point}

The RT neighbours [7] of a point $\mathrm{p}(\mathrm{x}, \mathrm{y})$ consists of the neighbours $(x-1, y+1)$ and $(x+1, y-1)$. A point ' $p$ ' and its RT neighbours is denoted by $\mathrm{R}_{3}(\mathrm{p})$.

\section{DEFINITIONS OF VARIOUS FUZZY HYBRID FILTERING TECHNIQUES}

In this section, the definitions of various fuzzy hybrid filters are given. The image processing function in a spatial domain can be expressed as

$$
g(p)=\mathrm{Y}(f(p))
$$

where $Y$ is the transformation function, $f(p)$ is the pixel value (intensity value or grey level value) of the point $\mathrm{p}(\mathrm{x}, \mathrm{y})$ of input image and $g(p)$ is the pixel value of the corresponding point of the processed image.

\subsection{Definition of Fuzzy filters [12]:}

Let $f(p)$ be the input image of a two dimensional fuzzy filter, the output the fuzzy filter is defined as :

$$
g(p)=\frac{\sum_{p \in N_{8}(p)} F(p) \cdot f(p)}{\sum_{p \in N_{8}(p)} F(p)}
$$

where $F(p)$ is the general 8-neighbour function. With the definition of fuzzy filters various fuzzy hybrid filtering techniques are defined, which we shall describe as Fuzzy hybrid max filter $\left(\mathrm{FH}_{3} \mathrm{~F}\right)$, the Gaussian fuzzy hybrid filter with hybrid max center(GFHF), the symmetrical triangular fuzzy hybrid filter with hybrid cross median center (TFHF) and the asymmetrical triangular fuzzy hybrid filter with hybrid max center(ATFHF).

\section{2 $\mathrm{FH}_{3} \mathrm{~F}$}

In fuzzy hybrid max filter, the general 8-neighbour function is defined as:

$$
F(p)=\left\{\begin{array}{lc}
1 & \text { for } f(p)=h m v(p), \mathrm{p} \in \mathrm{N}_{8}(\mathrm{p}) \\
0 & \text { otherwise }
\end{array}\right\}
$$

where $h m v(p)$ is the hybrid max value, which is the maximum of median pixel value of $L T$ neighbours of a point ' $p$ ', median pixel value of $R T$ neighbours of a point ' $p$ ' and pixel value of ' $p$ '.

\subsection{GFHF}

The Gaussian fuzzy hybrid filter with the hybrid max value within 8-neighbour of a point chosen as the center value is defined as:

$$
F(p)=e^{\frac{-1}{2}\left(\frac{f(p)-h m v(p)}{\sigma(p)}\right)^{2}}, \text { for } \mathrm{p} \in \mathrm{N}_{8}(\mathrm{p})
$$

where $h m v(p)$ and $\sigma(p)$ represents respectively, the hybrid max value and standard deviations all the input values of ' $p$ ' for $\mathrm{p} \in \mathrm{N}_{8}(\mathrm{p})$.

\subsection{TFHF}

The symmetrical triangular fuzzy hybrid filter with hybrid cross median value within 8-neighbour of a point chosen as the center value is defined as:

$F(p)=$

$\left\{\begin{array}{rr}1-\frac{|f(p)-h c m v(p)|}{n m(p)}, & \text { for }|f(p)-h c m v(p)| \leq n m(p) \\ 1 & \text { for } n m(p)=0\end{array}\right\}$ $n m(p)=\max [m x(p)-h c m v(p), h c m v(p)-m n(p)]$

where $\operatorname{hcmv}(\mathrm{p})$, the hybrid cross median value, which is the median of median pixel value of $L T$ neighbours of a point ' $p$ ', median pixel value of $R T$ neighbours of a point ' $p$ ' and pixel value of ' $p$ '. $m x(\mathrm{p})$ and $m n(\mathrm{p})$ are, respectively, maximum and minimum value of all the input values of ' $p$ ' for $p \in N_{8}(p)$.

\subsection{ATFHF}

The asymmetrical triangular fuzzy hybrid filter with hybrid max value within 8-neighbour of a point chosen as the center value is defined as:

$F(p)=$

$$
\left\{\begin{array}{c}
1-\frac{h m v(p)-f(p)}{h m v(p)-m n(p)}, \text { for } m n(p) \leq f(p) \leq h m v(p) \\
1-\frac{f(p)-h m v(p)}{m x(p)-h m v(p)}, \text { for } h m v(p) \leq f(p) \leq m x(p) \\
1, \operatorname{for} h m v(p)-m n(p)=0 \text { or } m x(p)-h m v(p)=0
\end{array}\right\}
$$

where $h m v(p)$ is the hybrid max value, which is the maximum of median pixel value of $L T$ neighbours of a point ' $p$ ', median pixel value of $R T$ neighbours of a point ' $p$ ' and pixel value of ' $p$ '. $m x(p)$ and $m n(p)$ are, respectively, maximum and minimum value of all the input values of ' $p$ ' for $p \in N_{8}(p)$.

\section{EXPERIMENTAL RESULT ANALYSIS AND DISCUSSION}

The proposed fuzzy hybrid filtering techniques have been implemented using MATLAB 7.0. The performance of the various fuzzy hybrid filtering techniques is analyzed and discussed. The measurement of noise reduction is difficult and there is no unique algorithm available to measure noise reduction of ultrasound images. So we use statistical tool to measure the noise reduction of ultrasound images. The Root Mean Square Error (RMSE) and Peak Signal-to-Noise (PSNR) are used to evaluate the enhancement of ultrasound images.

$$
\begin{aligned}
& \text { RMSE }=\sqrt{\frac{\sum(f(i, j)-g(i, j))^{2}}{m n}} \\
& \text { PSNR }=20 \log _{10} \frac{255}{R M S E} .
\end{aligned}
$$

Here $f(i, j)$ is the pixel value of original ultrasound image , $g(i, j)$ is the pixel value of filtered ultrasound image and $m$ and $\mathrm{n}$ are the total number of pixels in the horizontal and the vertical dimensions of the image. If the value of RMSE is low and value of PSNR is high then the noise reduction approach is better. The noisy ultrasound image of prostate image with various noise level of Gaussian noise of variance 0.0052, 0.021 and 0.106 . and filtered ultrasound image obtained by the various fuzzy hybrid filtering techniques. Table 4.1 shows the proposed fuzzy hybrid filtering techniques that are compared with some existing filtering techniques namely, MED, GMED, TMED, ATMED, MAV, GMAV, TMAV, ATMAV, DWMAV1, DWMAV2 and DWMAV3 with regard to ultrasound medical images for prostate image. 
Table 4.1: RMSE and PSNR values for filtered image of variance 0.0052, 0.021 and .106 .

\begin{tabular}{|c|c|c|c|c|c|c|c|c|c|}
\hline \multirow{2}{*}{\multicolumn{2}{|c|}{ Filters }} & \multicolumn{3}{|c|}{ Variance of Random Noise } & \multirow{2}{*}{\multicolumn{2}{|c|}{ Filters }} & \multicolumn{3}{|c|}{ Variance of Random Noise } \\
\hline & & \multirow{2}{*}{\begin{tabular}{|c|} 
Low \\
$\mathbf{0 . 0 0 5 2}$
\end{tabular}} & \multirow{2}{*}{$\begin{array}{c}\text { Medium } \\
\mathbf{0 . 0 2 1} \\
1.4573 \\
\end{array}$} & \multirow{2}{*}{\begin{tabular}{c|} 
High \\
0.106 \\
1.9907 \\
\end{tabular}} & & & \multirow{2}{*}{\begin{tabular}{|c|} 
Low \\
$\mathbf{0 . 0 0 5 2}$
\end{tabular}} & \multirow{2}{*}{\begin{tabular}{|c|}
$\begin{array}{c}\text { Medium } \\
\mathbf{0 . 0 2 1}\end{array}$ \\
10.9424 \\
\end{tabular}} & \multirow{2}{*}{\begin{tabular}{|c|}
$\begin{array}{c}\text { High } \\
\text { 0.106 }\end{array}$ \\
12.8673
\end{tabular}} \\
\hline \multirow{2}{*}{$\mathrm{FH}_{3} \mathrm{~F}$} & RMSE & & & & \multirow{2}{*}{ MAV } & RMSE & & & \\
\hline & PSNR & 45.5893 & 44.8607 & 42.1511 & & PSNR & 27.6411 & 27.3489 & 25.9414 \\
\hline \multirow{2}{*}{ GFHF } & RMSE & 10.5389 & 10.9424 & 12.8673 & \multirow{2}{*}{ GMAV } & RMSE & 10.55 & 10.9497 & 12.8995 \\
\hline & PSNR & 27.6752 & 27.3489 & 25.9414 & & PSNR & 27.6661 & 27.3431 & 25.9197 \\
\hline \multirow{2}{*}{ TFHF } & RMSE & 10.5611 & 10.9546 & 12.5545 & \multirow{2}{*}{ TMAV } & RMSE & 10.8006 & 11.146 & 12.7554 \\
\hline & PSNR & 27.657 & 27.3392 & 26.1551 & & PSNR & 27.4622 & 27.1888 & 26.0173 \\
\hline \multirow{2}{*}{ ATFHF } & RMSE & 7.8778 & 8.3942 & 10.996 & \multirow{2}{*}{ ATMAV } & RMSE & 8.83 & 9.305 & 11.66 \\
\hline & PSNR & 30.2031 & 29.6516 & 27.3065 & & PSNR & 29.212 & 28.7568 & 26.7972 \\
\hline \multirow{2}{*}{ MED } & RMSE & 8.6696 & 8.7733 & 8.9285 & \multirow{2}{*}{ DWMAV1 } & RMSE & 9.0701 & 9.4112 & 11.1736 \\
\hline & PSNR & 29.3712 & 29.2679 & 29.1156 & & PSNR & 28.9789 & 28.6583 & 27.1673 \\
\hline \multirow{2}{*}{ GMED } & RMSE & 10.5406 & 10.9497 & 12.901 & \multirow{2}{*}{ DWMAV2 } & RMSE & 9.7284 & 10.1317 & 11.972 \\
\hline & PSNR & 27.6738 & 27.3431 & 25.9187 & & PSNR & 28.3703 & 28.0175 & 26.5678 \\
\hline \multirow{2}{*}{ TMED } & RMSE & 10.8218 & 11.1737 & 12.7987 & \multirow{2}{*}{ DWMAV3 } & RMSE & 9.993 & 10.3909 & 12.2452 \\
\hline & PSNR & 27.4451 & 27.1672 & 25.9878 & & PSNR & 28.1372 & 27.7981 & 26.3718 \\
\hline \multirow{2}{*}{ ATMED } & RMSE & 9.4387 & 9.7584 & 11.7583 & & & & & \\
\hline & PSNR & 28.6329 & 28.3436 & 26.7242 & & & & & \\
\hline
\end{tabular}

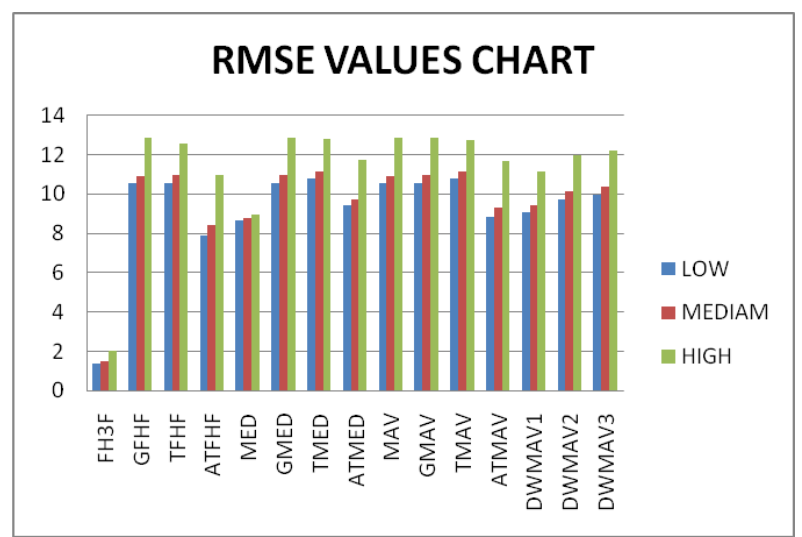

Figure 1: RMSE values

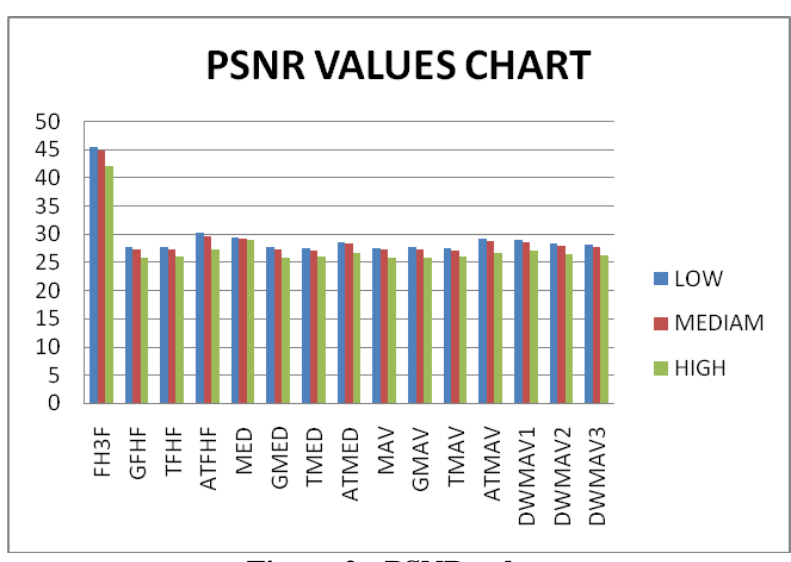

Figure 2 : PSNR values 


\section{CONCLUSION}

In this work, various fuzzy hybrid filtering techniques for removal of Gaussian noise from ultrasound medical images are introduced. To demonstrate the performance of the proposed techniques, the experiments have been conducted on ultrasound prostate images and compared our methods with other well known techniques. The experimental results indicate that the one of the proposed fuzzy hybrid max filter $\left(\mathrm{FH}_{3} \mathrm{~F}\right)$ performs significantly better than other existing techniques and it gives the best results. The proposed method is simple and easy to implement. These filters may be applied by researchers to reduce random noise in the ultrasound medical images.

\section{REFERENCES}

[1] Tukey J.W, Nonlinear (nonsuperposable) methods for smoothing data, in Proc. Congr. Rec. EASCOM '74, (1974),673-681Tavel, P. 2007 Modeling and Simulation Design. AK Peters Ltd.

[2] Kwan H. K and Cai Y.,Median filtering using fuzzy concept, Proceedings of $36^{\text {th }}$ Midwest Symposium on Circuits and Systems, Vol.2, (1993),824-827.

[3] Kwan, H.K., Cai Y., Fuzzy filters for image filtering, presented at Midwestern Symposium on Circuits and Systems, (2002),III672-III675.

[4] Nachtegael M,Van der Weken D, Van De Ville A, Derre E, Philips W, Lemahieu I, An overview of fuzzy filters for noise reduction, Proceedings of IEEE International conference on Fuzzy systems, (2001),7-10.

[5] Nachtegael M,Van der Weken D, Van De Ville A, Derre E, Philips W, Lemahieu I, A comparative study of classical and fuzzy filters for noise reduction,
Proceedings of IEEE International conference on Fuzzy systems, (2001),11-14.

[6] Schulte, S., Nachtegael, M., De Witte, V., Van der Weken, D., Kerre, E.E., A Fuzzy Impulse Noise Detection and Reduction Method. IEEE Transactions on Image Processing 15(5), (2006),1153-1162.

[7] Gnanambal Ilango and Marudhachalam R, New hybrid filtering techniques for removal of Gaussian noise from medical images, ARPN Journal of Engineering and Applied Sciences, Vol 6, No.2, (2011),8-12.

[8] Aneesh Agrawal, Abha Choubey and Kapil Kumar Nagwanshi, Development of adaptive fuzzy based Image Filtering techniques for efficient Noise Reduction in Medical Images, International Journal of Computer Science and Information Technologies Vol 2(4),(2011),1457-1461

[9] Gunamani Jena and $\mathrm{R}$ Baliarsingh, Suppression of Random Valued Impulsive Noise using Adaptive Threshold, International Journal of Latest Trends in Computing Vol.1.(2010),116-120.

[10] Rosenfeld A, Digital topology, Amer. Math. Monthly 86, (1979),621-630.

[11] Gonzalez. R and Woods. R, Digital Image Processing, Adison -Wesley, New York(1992).

[12] Kerre E E and Nachtegael M. Editors, Fuzzy Techniques in image processing, Series on Studies in Fuzziness and Soft Computing, Vol.52, (2000), Springer-Verlog. 